\title{
SHOCKS VIOLATING LAX'S CONDITION ARE UNSTABLE
}

\author{
E. D. CONWAY AND J. A. SMOLLER ${ }^{1}$
}

Abstract. For a system of two conservation laws, we prove that solutions violating Lax's condition across shocks are unstable relative to smoothing of the initial data.

In this note we show, for a hyperbolic system of two conservation laws, that weak solutions which violate the Lax shock conditions [3] are unstable relative to smoothing of the initial data. Our result is seen to be a consequence of the work of Johnson [2], and Yamaguti-Nishida [5].

We consider the system

$$
u_{t}+(\partial / \partial x) f(u, v)=0, \quad v_{t}+(\partial / \partial x) g(u, v)=0
$$

where $f$ and $g$ are assumed to be $C^{2}$ functions. Our context is that of Lax [3], Smoller and Johnson [4] and Glimm and Lax [1]. Our notation is that of [1] and [4], i.e., (1) is assumed to be strictly hyperbolic, genuinely nonlinear and to satisfy the shock interaction conditions. More precisely, we assume that $f_{v}<0, g_{u}<0$, and that under the normalizations given in [4] $, l_{i} d^{2} F\left(r_{j}, r_{j}\right)>0, i, j=1,2$. Here $l_{i}$ and $r_{j}$ are the left and right eigenvectors, respectively of $d(f, g)$, the Jacobian matrix associated with the mapping $F=(f, g)$. In terms of the Riemann invariants $w, z$, the system (1) takes the form

$$
w_{t}+\rho w_{x}=0, \quad z_{t}+\lambda z_{x}=0,
$$

where $\lambda$ and $\rho$ are the eigenvalues of $d F, \lambda>\rho$.

Because of the finite speed of propagation, it is sufficient to prove instability for solutions having only one shock. For simplicity, we also assume it to be piecewise constant. Therefore, we consider solutions of the form

$$
\begin{aligned}
(u(t, x), v(t, x)) & =\left(u_{0}, v_{0}\right), & & x<s t, \\
& =\left(u_{1}, v_{1}\right), & & x>s t,
\end{aligned}
$$

where $s$ is the (constant) shock speed. For such a function to be a (weak)

Received by the editors September 27, 1972.

AMS (MOS) subject classifications (1970). Primary $35 \mathrm{~L} 40$.

${ }^{1}$ Sponsored by the United States Army under Contract No. DA-31-124-ARO-D-462.

(c) American Mathematical Society 1973 
solution of (1), it is necessary and sufficient that the following jump relations be satisfied:

$$
s\left(u_{1}-u_{0}\right)=f\left(u_{1}, v_{1}\right)-f\left(u_{0}, v_{0}\right), \quad s\left(v_{1}-v_{0}\right)=g\left(u_{1}, v_{1}\right)-g\left(u_{0}, v_{0}\right),
$$

or if we eliminate $s$,

(4) $\left(v_{1}-v_{0}\right)\left(f\left(u_{1}, v_{1}\right)-f\left(u_{0}, v_{0}\right)\right)=\left(u_{1}-u_{0}\right)\left(g\left(u_{1}, v_{1}\right)-g\left(u_{0}, v_{0}\right)\right)$.

If we keep $\left(u_{0}, v_{0}\right)$ fixed, then the set of states $\left(u_{1}, v_{1}\right)$ which satisfy (4) form two smooth curves intersecting at $\left(u_{0}, v_{0}\right)$ (cf. [4], for example). Figure 1 below denotes a typical situation. Shocks in which $\left(u_{1}, v_{1}\right)$ lies on either of two of the four branches, denoted by $S_{1}$ and $S_{2}$ in Figure 1,
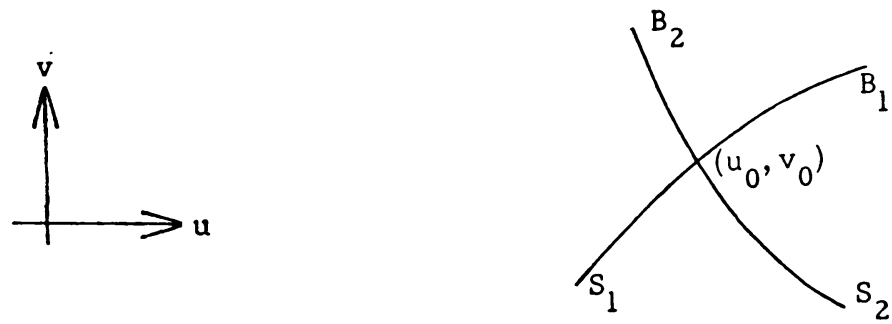

Figure 1

satisfy the Lax shock condition, whereas if $\left(u_{1}, v_{1}\right)$ lies on $B_{1}$ or $B_{2}$, the Lax shock condition is violated [4]. Our result is that shocks in which $\left(u_{1}, v_{1}\right)$ lies on $B_{1}$ or $B_{2}$ are unstable relative to smoothing. The key ingredient is provided by the following lemma.

Lemma. If $\left(u_{1}, v_{1}\right)$ lies on $B_{1}$ or $B_{2}$, then $w\left(u_{1}, v_{1}\right)>w\left(u_{0}, v_{0}\right)$, and $z\left(u_{1}, v_{1}\right)>z\left(u_{0}, v_{0}\right)$.

We postpone the proof of the lemma until the end of the paper.

Now let $U(t, x)=(u(t, x), v(t, x))$, where $u$ and $v$ are as in (3), with $\left(u_{1}, v_{1}\right)$ on $B_{1}$ or $B_{2}$. We show instability of $U$ by showing that for certain smooth functions $U_{n}(0, x)$ converging to $U(0, x)$, the equations (1) have smooth solutions which are defined for all $(t, x), t \geqq 0$, and which converge not to $U$, but to a Lipschitz continuous solution of (1).

Let $R(t, x)=(w(t, x), z(t, x))$ be the Riemann invariant form of the solution $U$. From the lemma we see that $w^{0}(x)=w(0, x)$ and $z^{0}(x)=z(0, x)$ are nondecreasing functions; in fact, they are step functions having one upward jump at $x=0$. Let $\left\{w_{n}^{0}\right\}$ and $\left\{z_{n}^{0}\right\}$ be sequences of smooth functions converging uniformly to $w^{0}$ and $z^{0}$ on compact sets not containing $x=0$. The corresponding functions $u_{n}^{0}$ and $v_{n}^{0}$ similarly converge to $u(0, x)$ and $v(0, x)$. As shown by Yamaguti and Nishida in [5], the equations (2) have 
smooth solutions $\left(w_{n}, z_{n}\right)$ defined for all $t>0$, which moreover satisfy $\left(w_{n}(0, x), z_{n}(0, x)\right)=\left(w_{n}^{0}(x), z_{n}^{0}(x)\right)$. Furthermore $\left\{\left(w_{n}, z_{n}\right)\right\}$ converges uniformly on compact sets to functions $(\bar{w}(t, x), \bar{z}(t, x))$ which are locally Lipschitz continuous in $t>0$, satisfy (2) almost everywhere in $t>0$, and $(\bar{w}(0, x), \bar{z}(0, x))=\left(w^{0}(x), z^{0}(x)\right)=(w(0, x), z(0, x))$. This proves the instability of the solution $U$.

Proof OF THE Lemma. We shall only give the proof for "back" shocks; i.e., shocks associated with the smaller characteristic family, $\lambda$; the proof for "front" shocks is similar. Thus we assume that (3) represents a back shock, and we must prove that $w\left(u_{1}, v_{1}\right)>w\left(u_{0}, v_{0}\right)$, and $z\left(u_{1}, v_{1}\right)>z\left(u_{0}, v_{0}\right)$.

The strength of the (back) shock (3) is defined to be

$$
\varepsilon_{1}=w\left(u_{1}, v_{1}\right)-w\left(u_{0}, v_{0}\right) .
$$

Now from [3], we know that along $B_{1}, \varepsilon_{1}>0$, so that $w\left(u_{1}, v_{1}\right)>w\left(u_{0}, v_{0}\right)$. Next, from [4], we have

$$
z\left(u_{1}, v_{1}\right)-z\left(u_{0}, v_{0}\right)=\left(\frac{1}{12}\right)(\lambda-\rho)^{-2} l_{2} d^{2} F\left(r_{1}, r_{1}\right) \varepsilon_{1}^{3}+O\left(\varepsilon_{1}^{4}\right),
$$

so that under our hypotheses, we see that $z\left(u_{1}, v_{1}\right)>z\left(u_{0}, v_{0}\right)$ for small $\varepsilon_{1}>0$. Hence the curve $B_{1}$ starts out in the desired region, cf. Figure 2.
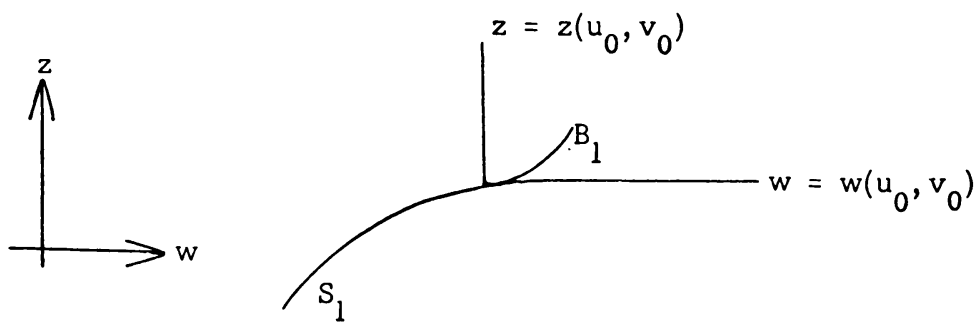

FIGURE 2

If $B_{1}$ were to ever leave this region, then since $w$ always increases along $B_{1}$, there must be a first point $(u, v)$, different from $\left(u_{0}, v_{0}\right)$, where $z(u, v)=0$. Since the curves $z=$ const are integral curves of a vector field, they cannot
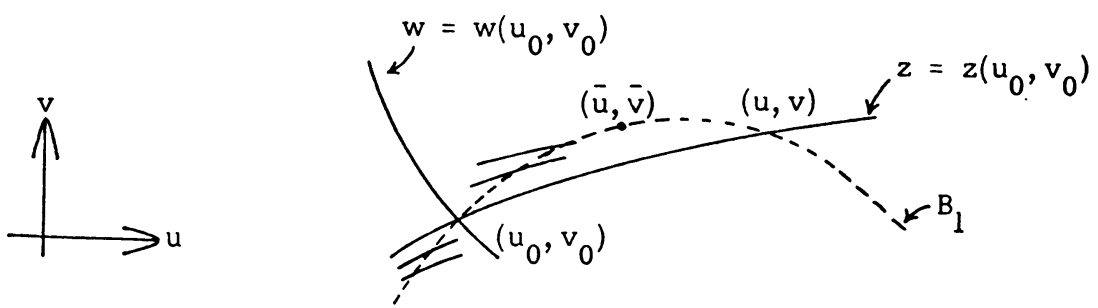

Figure 3 
intersect each other. Thus there is a point $(\bar{u}, \bar{v})$ on $B_{1}$ where the slope of the curve $z=z(\bar{u}, \bar{v})$ equals the slope of $B_{1}$ at $(\bar{u}, \bar{v})$ (cf. Figure 3 , where the curves of positive slope cutting $B_{1}$ transversally are the curves $z=$ const). In the notation of [4], we have $a_{1}(\bar{u}, \bar{v})=h_{1}(\bar{u}, \bar{v})$. But, just as in [4, Theorem 2.3], we have, for back shocks, $h_{1}>a_{1}$, all along $B_{1}$. This is a contradiction, and the proof of the lemma is complete. (Alternatively, if $h_{1}(\bar{u}, \bar{v})=a_{1}(\bar{u}, \bar{v})$, then it is not too hard to show that $\lambda(\bar{u}, \bar{v})=s(\bar{u}, \bar{v})$, but just as in [4, Theorem 2.2], we have $\lambda>s$ all along this branch.)

\section{REFERENCES}

1. J. Glimm and P. D. Lax, Decay of solutions of systems of nonlinear hyperbolic conservation laws, Mem. Amer. Math. Soc. No. 101 (1970). MR 42 \#676.

2. J. L. Johnson, Global continuous solutions of hyperbolic systems of quasi-linear equations, Bull. Amer. Math. Soc. 73 (1967), 639-641. MR 35 \#4593.

3. P. D. Lax, Hyperbolic systems of conservation laws. II, Comm. Pure Appl. Math. 10 (1957), 537-566. MR 20 \#176.

4. J. A. Smoller and J. L. Johnson, Global solutions for an extended class of hyperbolic systems of conservation laws, Arch. Rational Mech. Anal. 32 (1969), 169-189; Erratum, ibid. 37 (1970), 399-400. MR 38 \#4822; MR 41 \#645.

5. M. Yamaguti and T. Nishida, On some global solution for quasi linear hyperbolic equations, Funkcial. Ekvac. 11 (1968), 51-57. MR 39 \#634.

Department of Mathematics, Tulane University, New Orleans, louisiana 70118 (Current address of E. D. Conway)

Department of Mathematics, University of Michigan, Ann Arbor, Michigan 48104

Current address (J. A. Smoller): Department of Mathematics, University of Wisconsin, Madison, Wisconsin 53706 